\title{
miR-205 targets angiogenesis and EMT concurrently in anaplastic thyroid carcinoma
}

\author{
Haleh Vosgha', Armin Ariana', Robert Anthony Smith ${ }^{1,2}$ and Alfred King-yin Lam ${ }^{1}$ \\ ${ }^{1}$ Cancer Molecular Pathology, School of Medicine, Menzies Health Institute Queensland, Griffith University, Gold Coast, Queensland, Australia \\ ${ }^{2}$ Genomics Research Centre, Institute of Health and Biomedical Innovation, Faculty of Health, Queensland University of Technology, Brisbane, \\ Queensland, Australia \\ Correspondence should be addressed to A Ariana or A Lam: a.ariana@griffith.edu.au or a.lam@griffith.edu.au
}

\begin{abstract}
The current study aims to evaluate for the first time the inhibitory roles of miR-205 in the pathogenesis of anaplastic thyroid carcinoma. In addition, we investigated the mechanisms by which miR-205 regulates angiogenesis and epithelial-to-mesenchymal transition (EMT) in cancer. Two anaplastic thyroid carcinoma cell lines were transfected with the expression vector PCMV-MIR-205. Selected markers of angiogenesis and EMT including vascular endothelial growth factor $A(V E G F-A)$ and zinc finger E-box-binding homeobox 1 (ZEB1) were investigated by Western blot. The interaction of miR-205 expression with EMT and angiogenesis were also investigated by assessment of matrix metalloproteinases 2 and 9 (MMP2 and MMP 9), SNAl1 (Snai1 family zinc finger 1), vimentin, E-cadherin and N-cadherin. The function of miR-205 was further tested with VEGF enzyme-linked immunosorbent assay (ELISA), wound healing, invasion and tube formation assays. Using an animal model, we studied the association of miR-205 with angiogenesis, proliferation and invasion. The following results were obtained. Permanent overexpression of miR-205 significantly suppressed angiogenesis and EMT by simultaneously targeting VEGF-A, ZEB1 and downstream products. Ectopic expression of miR-205 in cancer cells led to decreased migration, invasion and tube formation of endothelial cells. In addition, inhibition of tumour growth, vascularisation and invasion were noted in the mouse tumour xenografts. Our findings provide insights into simultaneous regulatory role of miR-205 in the pathogenesis of anaplastic thyroid carcinoma by suppressing both angiogenesis and EMT. This may open avenues to exploit miR-205 as an alternative cancer therapeutic strategy in the future.
\end{abstract}

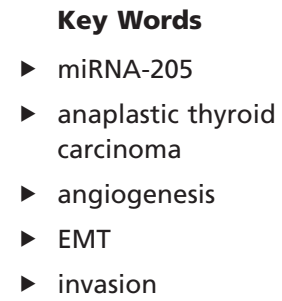

Endocrine-Related Cancer (2018) 25, 323-337

\section{Introduction}

Anaplastic thyroid carcinoma is one of the most aggressive and chemotherapy-resistant types of all thyroid carcinomas. The majority of patients with anaplastic thyroid carcinoma die within a year of diagnosis (Lo et al. 1999, Lam et al. 2000). In the current World Health Organization (WHO) classification of endocrine tumours, it is acknowledged that the genetic mechanisms and molecular profiles in the development and progression of anaplastic thyroid carcinoma is complex, which is consistent with the aggressive nature of this lethal malignancy (Lam 2017). Amongst the molecular changes occurring in anaplastic thyroid carcinoma development, angiogenesis and epithelialto-mesenchymal transition (EMT) are important 
factors in overall pathogenesis (Braun et al. 2010, Giatromanolaki et al. 2010).

Angiogenesis is the process of the development of new blood vessels, which facilitates cancer growth and metastases (Folkman \& Shing 1992). In this process, hypoxia plays an important role in activating the proteins and pro-angiogenic factors involved (Fig. 1). Vascular endothelial growth factor A $(V E G F-A)$, above all other known pro-angiogenic factors, induces sprouting of new blood vessels. In thyroid carcinomas, VEGF- $A$ is the principal regulator of angiogenesis (Salajegheh et al. 2013). In many cancers, the roles of matrix metalloproteinase 2 (MMP2) and matrix metalloproteinase 9 (MMP9) in dissolving extracellular matrix (ECM) and assisting and promoting angiogenesis have been suggested (Sang 1998, Hoeben et al. 2004, Araujo et al. 2015). The interactions of these proteins and their potential roles in the invasive behaviour of anaplastic thyroid carcinoma are yet to be addressed properly (Alfano et al. 2010, Jia et al. 2013, Wajner et al. 2014).

EMT as a crucial process for cancer metastasis starts at the earliest stages of tumour progression. The process leads to the loss of epithelial-specific characteristics, cell-cell adhesions, gain of stem-like properties of cancer cells and a migratory phenotype (van Zijl et al. 2011, De Craene \& Berx 2013). Well-described EMT inducer factors like ZEB1 (zinc finger E-box-binding homeobox 1), SNAI1 (snai1 family zinc finger 1 ), $\mathrm{N}$-cadherin and vimentin govern EMT through repression of epithelial markers like E-cadherin (Tsai \& Yang 2013). Interestingly, there is a link between angiogenesis and EMT-induced cancer cell stemness, which can activate tumorigenicity and malignant transformation mechanisms (Fantozzi et al. 2014). In addition, ZEB1 overexpression has been correlated with increased formation of new blood vessels and tumourigenesis through the induction of VEGF-A expression (Liu et al. 2016). Hypoxia is also one of the main inducers of EMT by upregulation of Snai1, ZEB1/2, Twist, vimentin and N-cadherin (Fig. 1) (Salnikov et al. 2012, De Craene \& Berx 2013, Lamouille et al. 2014).

In thyroid carcinomas, several miRNAs have been used to predict the clinical aggressiveness and the prognosis of patients with cancer (Chruscik \& Lam 2015). miRNA205-5p ( $m i R-205)$ is implicated in numerous signalling pathways, in particular angiogenesis and EMT (Qin et al. 2013, Vosgha et al. 2014). miR-205 plays a central role in tumour vascularisation as well as tumour invasion through targeting $V E G F-A$ and $Z E B 1$ in cancers including melanoma, glioblastoma, ovarian carcinoma and breast carcinoma (Fig. 1) (Gregory et al. 2008, Xu et al. 2012, Yue et al. 2012, Zhang et al. 2014, Li et al. 2015a).

Induction of miR-205 expression could potentially be used as an anti-angiogenic method to inhibit VEGF-A expression and tumour angiogenesis in thyroid carcinoma (Salajegheh et al. 2015). As there is a common resistance to current conventional therapies by anaplastic thyroid carcinoma, miRNA-targeted therapy may offer hope to develop molecular therapeutic approaches for patients

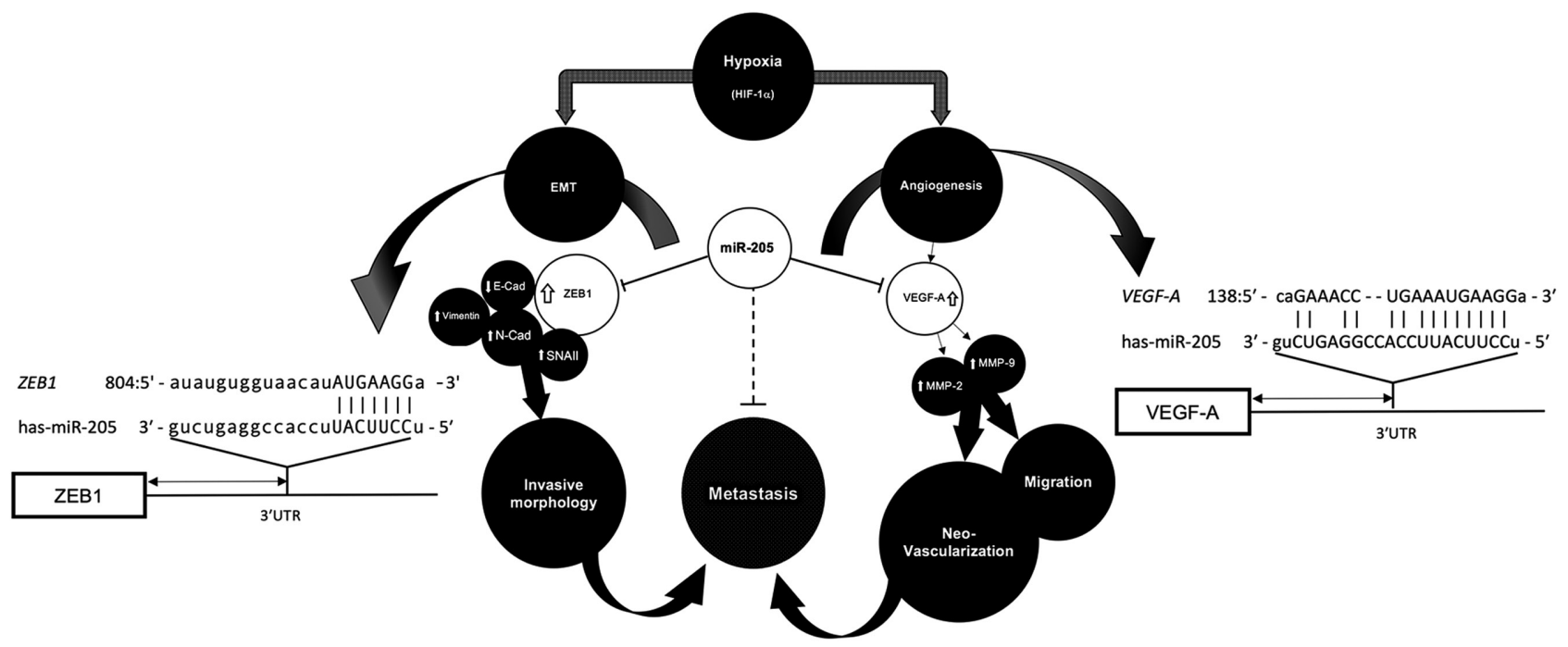

Figure 1

miR-205 targets VEGFA, ZEB1 and potentially other cross-talking EMT markers. Schematic diagram of hypoxic-induced angiogenesis and EMT, and potential role of miR-205 in the inhibition of vascularisation and invasion by targeting VEGFA and ZEB1. Schematic diagram illustrating predicted target sites of miR-205 within the 3'UTR of VEGFA and ZEB1. 
with this cancer. Therefore, the aim of this research was to take advantage of the capability of miR-205 to impede angiogenic and EMT mechanisms as two powerful events in the pathogenesis of thyroid cancer. In this study, inhibition of pathways of angiogenesis and metastasis by partially targeting the involved genes of these pathways were examined and demonstrated after permanent re-introduction of miR-205 in vitro and in vivo.

\section{Materials and methods}

\section{Cell culture}

MB-1 (ACC638, from a human anaplastic thyroid carcinoma growing in the thyroid) and BHT-101 (ACC279, from a metastatic human anaplastic thyroid carcinoma growing in a lymph node) were purchased from Deutsche Sammlung von Mikroorganismen und Zellkulturen, German Collection of Microorganisms and Cell Cultures (DSMZ, Braunschweig, Germany). STR data for authenticity of the respective cell lines were generated by DNA profiling by the DSMZ Company. MB-1 cancer cells were cultured in 80\% Roswell Park Memorial Institute 1640 (RPMI-1640), 20\% fetal bovine serum (FBS) and $2 \mathrm{mM}$ L-glutamine. BHT-101 cells were maintained in 80\% Dulbecco's Modified Eagle Medium (DMEM), 20\% FBS and 0.5\% human serum. Both media contained $100 \mathrm{U} / \mathrm{mL}$ penicillin and $100 \mu \mathrm{g} / \mathrm{mL}$ streptomycin. For cell expansion and transfection manipulation, cells were incubated in a humidified incubator with $5 \% \mathrm{CO}_{2}$ and at $37^{\circ} \mathrm{C}$. When angiogenic events were studied, cells were incubated in hypoxic conditions $\left(1 \% \mathrm{O}_{2}\right)$ at $37^{\circ} \mathrm{C}$ to create similarity to the microenvironment of malignant lesions. Cancer cells that were sub-cultured more than 5 times were excluded in this study. In addition, an experienced scientist of the team preserved the authenticity of cells by routinely monitoring growth curves, cell morphology and absence of any mycoplasma contamination.

\section{Cell transfection}

pCMV-MIR-205 expression vector (OriGene, Rockville, $\mathrm{MD}$, USA) containing green fluorescent protein (GFP) as a reporter gene and the empty vector pCMV-MIR as a mock control vector were transfected using Lipofectamine 3000 (Life Technologies) following the manufacturer's instructions. Briefly, $2 \times 10^{5} \mathrm{MB} 1$ and BHT-101 cells were seeded in a 12-well plate to reach $70-90 \%$ confluency. DNA $(1 \mu \mathrm{g})$ - Lipofectamine $3000(3 \mu \mathrm{L})$ complex was diluted in Opti-MEM reduced serum medium (Life Technologies). They were gently mixed and incubated for $5 \mathrm{~min}$ at room temperature. A total of $100 \mu \mathrm{L}$ of vector and Lipofectamine was directly added to cells in a final volume of $1 \mathrm{~mL}$ per well. The cells were incubated for $24 \mathrm{~h}$ and monitored under a fluorescent microscope. This was performed to check the expression of GFP and transfection efficiency. Stable cell lines were created using $600 \mu \mathrm{g} / \mathrm{mL}$ Geneticin Selective antibiotic (G418 Sulfate) (Thermo Fisher Scientific).

\section{RNA extraction and quantitative real-time PCR (RT-PCR)}

Total RNA and miRNA from the MB1 and BHT-101 pCMV-MIR-205-transfected cells, pCMV-MIR mock and untransfected cells were extracted (RNeasy Mini Kit; Qiagen). cDNA was synthesised (Qiagen miScript Reverse Transcription kit). RT-PCR was performed to estimate the expression of miR-205 in selected cancer cells as described previously (Salajegheh et al. 2015, 2016).

\section{Western blot}

Total cell lysate collection and protein extraction were performed and the concentration was measured (Protein quantification assay Macherey-Nagel $\mathrm{GmbH}$ \& Co. KG, Düren, Germany), and $30 \mu \mathrm{g}$ of extracted protein from cell lysates were run and separated on a $4-15 \%$ precast polyacrylamide gel (Mini-PROTEAN TGX Precast Gel; Bio-Rad). The proteins were then transferred onto polyvinylidene difluoride membranes (Trans-Blot Turbo Mini PVDF Transfer Packs; Bio-Rad) using a blotting instrument (Trans-Blot Turbo Transfer Starter System; Bio-Rad). Blocking of the membrane was performed using 5\% milk in Tris-buffered saline and Tween $20(120 \mathrm{mmol} / \mathrm{L}$ Tris-HCl, pH 7.4, $150 \mathrm{mmol} / \mathrm{L}$ $\mathrm{NaCl}$ and $0.05 \%$ Tween 20: TBST) for $1 \mathrm{~h}$ at room temperature. The membrane was incubated with antibodies overnight at $4^{\circ} \mathrm{C}$. The antibodies used were VEGF-A (sc-152, a rabbit polyclonal antibody at 1:100 concentration), ZEB1 (sc-81428, a mouse monoclonal antibody at 1:200 concentration), MMP2 (sc-13594, a mouse monoclonal antibody at 1:200 concentration), MMP9 (sc-21733, a mouse monoclonal antibody at 1:200 concentration), E-cadherin (sc-7870, a rabbit polyclonal antibody at 1:200 concentration), $\mathrm{N}$-cadherin (sc-393933, a mouse monoclonal antibody at 1:200 concentration), vimentin (sc-7557, a goat polyclonal antibody at 1:200 concentration) and SNAI1 (21-35, 
a rabbit polyclonal antibody at 1:500 concentration). VEGF-A, ZEB1, MMP2, MMP9, E-cadherin, N-cadherin and vimentin were obtained from Santa Cruz (Santa Cruz Biotechnology), whereas SNAI1 was obtained from Sigma-Aldrich. Following antibody incubation, the membrane was washed three times with TBST. Then, it was incubated with the secondary antibody conjugated to horseradish peroxidase for $1 \mathrm{~h}$. Signals were developed using the Western Lighting Plus-ECL substrate (Bio-Rad). ImageStudio (LI-COR, Cambridge, UK) was used for image analyses and densitometry measurement.

\section{Wound healing}

To determine the cell migration ability, a wound-healing assay was performed. pCMV-MIR-205-transfected cells, pCMV-MIR mock and untransfected cells were cultured overnight. After making the scratch with a p200 pipette tip, the wound area was measured within three days in the transfected and control groups. The cells were gently washed with $1 \times$ phosphate buffer saline (PBS) two times and then incubated with media containing reduced FBS. This was to confirm that the results were based on the migratory ability of the cancer cells, not their proliferation (starvation was optimised at 5\%). The images of wound areas were captured after 24,48 and $72 \mathrm{~h}$ by an inverted microscope at $10 \times$ magnification. Three different fields per well were selected randomly and captured. The mean wound area between one side of scratch to the other side was calculated and analysed using ImageJ software (Version 1.51; http://imagej.nih.gov/ij/).

\section{Cell invasion assay}

MB1 and BHT-101 pCMV-MIR-205-transfected cells, pCMV-MIR mock and untransfected cells were incubated in transwell inserts (Corning) with $8-\mu \mathrm{m}$ pore size and $6.5-\mathrm{mm}$ polycarbonate membranes coated with $0.3 \mathrm{mg} / \mathrm{mL}$ matrigel (ECM gel, Sigma-Aldrich) diluted in $50 \mu \mathrm{L}$ serumfree media. $2 \times 10^{5}$ pCMV-MIR-205-transfected cells, pCMV-MIR mock and untransfected cells in $100 \mu \mathrm{L}$ serumfree media cells were seeded into the upper compartment of the transwell chambers, and $650 \mu \mathrm{L}$ complete media was added to the lower chambers. After $24 \mathrm{~h}$, the number of invading cells were calculated and compared in these groups. The images of five random locations from three replicates of each transwell insert were captured using 10x magnification of microscope. The mean number of invasive cells for each condition was then measured and calculated using ImageJ software.

\section{ELISA assay}

The amount of VEGF-A protein concentration in the extracellular medium (culture media) was measured by means of human VEGF-A-165 enzymelinked immunosorbent assay (ELISA) novex kit (Life Technologies). pCMV-MIR-205-transfected cells, pCMV-MIR mock and untransfected cells were seeded at the density of $5 \times 10^{5}$ cells $/ \mathrm{mL}$ into the $10 \mathrm{~cm}^{2}$ tissue culture dishes and incubated in hypoxic conditions $\left(1 \% \mathrm{O}_{2}\right)$ at $37^{\circ} \mathrm{C}$. Culture media were then collected after 24,48 and $72 \mathrm{~h}$, and VEGF absorbance was quantified with a PolarStar Omega microplate reader (BMG Labtech, Ortenberg, Germany) at a wavelength of $450 \mathrm{~nm}$ and according to kit instructions.

\section{Tube formation assay}

Transwell cell culture chambers (Corning) with polycarbonate filters ( $4 \mu \mathrm{m}$ pore size; $0.33 \mathrm{~cm}^{2}$ area) were used for a co-culture assay with human umbilical vein endothelial cells (HUVEC; Lonza, Basel, Switzerland) and cancer cells. Endothelial growth medium-2 (EGM-2) media was diluted with growth factor reduced matrigel (ECM gel, from Engelbreth-Holm-Swarm mouse sarcoma, Sigma) without VEGF at a final concentration of $1 \mathrm{mg} / \mathrm{mL}$. $1 \times 10^{5} / \mathrm{mL}$ cancer cells were seeded into the transwell inserts (upper chambers) and incubated for $24 \mathrm{~h}$ at hypoxic conditions $\left(1 \% \mathrm{O}_{2}\right) .3 \times 10^{4}$ HUVEC cells were suspended in $500 \mu \mathrm{L}$ VEGF-free media and added to each well pre-coated with matrigel (lower chambers) in triplicate. Then, the transwell inserts were transferred to the top of the HUVEC cells overnight. After incubation, the tube formation ability of endothelial cells was imaged using an inverted microscope $4 \times$ magnification. Wimasis WimTube (Wimasis GmbH, Munich, Germany) software was used to analyse the number of loops and number of branching points.

\section{Tumour xenografts studies in mice}

In order to perform xenograft studies, animal ethical approval was obtained from Griffith University Animal Ethics Committee in 2016 (MED/02/15/AEC). Six-toeight-week-old female NU/NU nude mice were purchased through the animal facility at Griffith University. They were maintained in a well-ventilated cage in accordance with the Australian code for the care and use of animals for scientific purposes published by the National Health and Medical Research Council. $2 \times 10^{6}$ MB-1 
pCMV-MIR-205-transfected cells, pCMV-MIR mock cells and untransfected cells as well as $2 \times 10^{6}$ BHT-101 pCMV-MIR-205-transfected cells, pCMV-MIR mock and untransfected cells were suspended in cell media and matrigel (1:1). According to the Guidelines for the Welfare and Use of Animals in Cancer Research and the power calculator suggested in Workman et al. (2010), the population size was set to $80 \%$ power to produce significant results. Based on possible variations in the formation of new blood vessels within three experimental groups, 7 mice were entered into each group. The cancer cells were injected subcutaneously into the flanks of the nude mice. Tumours' growth in the mice were monitored and measured with a calliper ruler at different time points. The tumour volumes were also calculated using the formula: (width) $)^{2} \times$ length $\times 1 / 2$. After 28 days and when progressive tumour growth was evident, killing was performed. Resected tumour tissues were weighted (grams) and immersed in formaldehyde for paraffin embedding, sectioning and immunohistochemical staining.

\section{Immunohistochemical staining}

Resected tumour tissues were fixed in formalin and embedded in paraffin. Sections were cut from the tissues and stained with haematoxylin and eosin staining for histological examination by a pathologist (AKL). A marker of thyroid gland differentiation, thyroid transcription factor 1 (TTF1), was used to assess the differentiation of the thyroid cancer cells. Furthermore, the proliferative index, distribution of endothelial cells and EMT processes were evaluated by Ki-67, CD34, E-cadherin and vimentin staining respectively.

\section{Micro-vessel density quantification (MVD)}

Tumour-associated vessel formation was examined in samples stained with CD34 as the marker of endothelial cells. Assessment of micro-vessel density (MVD) in anaplastic thyroid carcinoma was performed by light microscopy. Positive staining of CD34 in endothelial cells was examined and identified by the pathologist of the team (AKL). In addition, the number of endothelial tubes formed in the tumour tissues were calculated and determined by counting five high-power $(\times 40)$ fields of the highest vascular density. MVD was expressed as the mean value of the micro-vessels counted in sum of those fields. We also considered any stained endothelial cell or clusters of endothelial cells separated from other microvessel structures as a countable micro-vessel.

\section{Data analysis}

All the experiments and assays were repeated in triplicates. The data retrieved from miRNA and protein expressions as well as the values measured by different imaging software and instruments described were entered into SPSS (statistical analysis in social science) software (version 24; IBM Corp.) for statistical analysis and Prism 3 GraphPad Software for graphs and charts demonstration. Final normalised data were analysed using paired and independent $t$-tests and analysis of variance (ANOVA), using Bonferroni and LSD correction. The significance threshold was taken at $P<0.05$. Finally, experimental results were demonstrated as mean values \pm S.E.M.

\section{Results}

Stable expression of $\mathbf{m i R}-205$ precursor in anaplastic thyroid carcinoma cell lines

After successful transfection of pCMV-MIR-205 expression vector and the empty vector pCMV-MIR, the level of miR-205 expression was evaluated by performing qRT-PCR. Significant overexpression of miR-205 was observed in both anaplastic thyroid carcinomas (MB-1 and BHT-101) compared with mock transfected and untransfected cancer cells $(P<0.05)$ (Fig. 2 A and B). This result confirmed the permanent ectopic expression of $m i R-205$ in the anaplastic carcinoma cells (MB-1 and BHT-101).

\section{miR-205 potentially acts against malignant angiogenesis and invasion in anaplastic thyroid carcinoma cell lines}

As shown in Fig. 1, VEGF- $A$ and $Z E B 1$ have been considered to be targets of $m i R-205$. In concordance with previous reports, we similarly observed repression of luciferase activity after co-transfection of VEGFA 3'-UTR and ZEB1 $3^{\prime}$-UTR into the luciferase reporter vector (not published) confirming that these sites have functions in anaplastic thyroid carcinoma cells (Gregory et al. 2008, Xu et al. 2012, Yue et al. 2012, Zhang et al. 2014, Li et al. 2015a).

Following confirmatory qRT-PCR experiments to show the ectopic expression of miR-205, Western blot was performed to check the expression of VEGF-A as a 

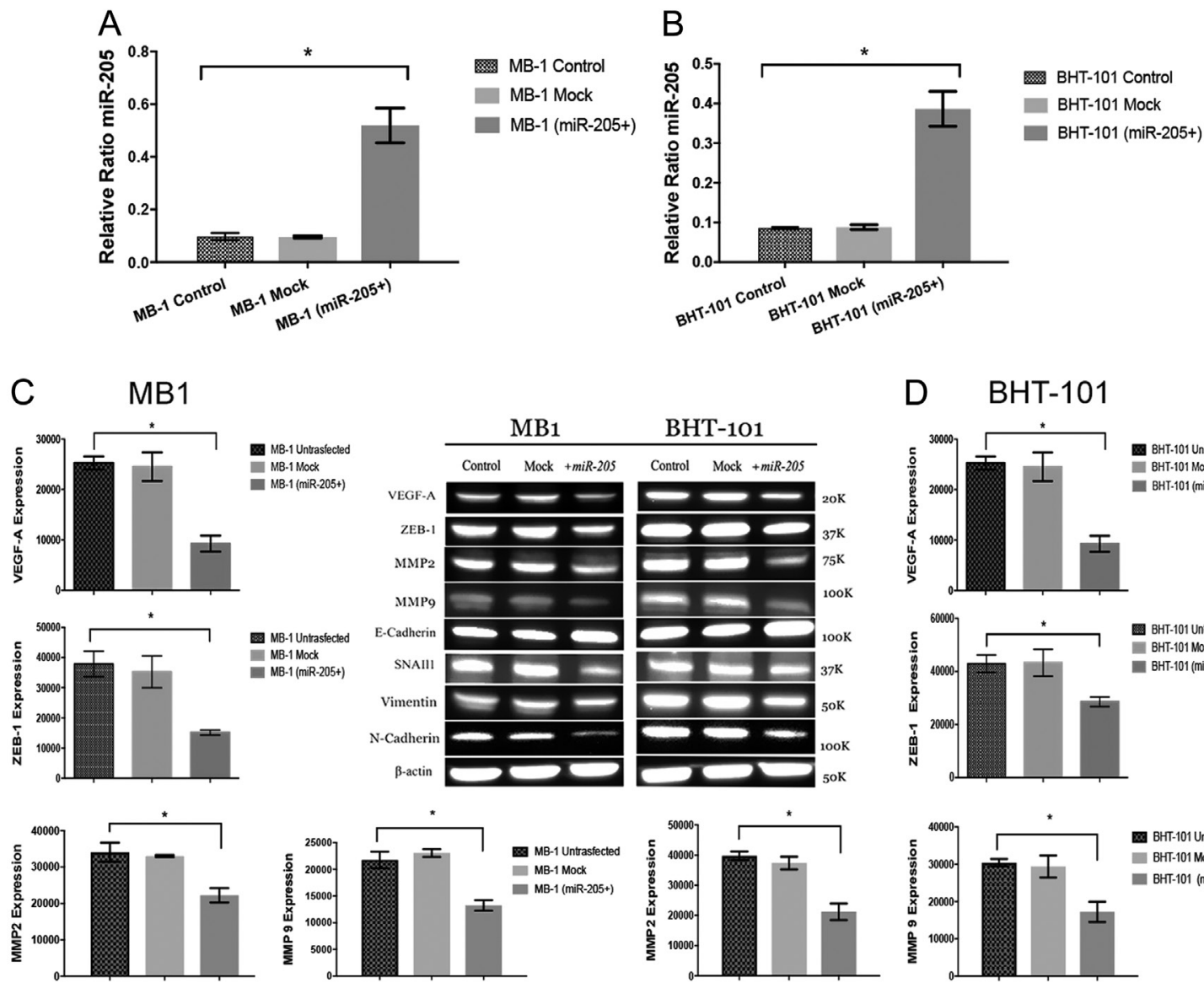

BHT-101

D $\quad$ BHT-101
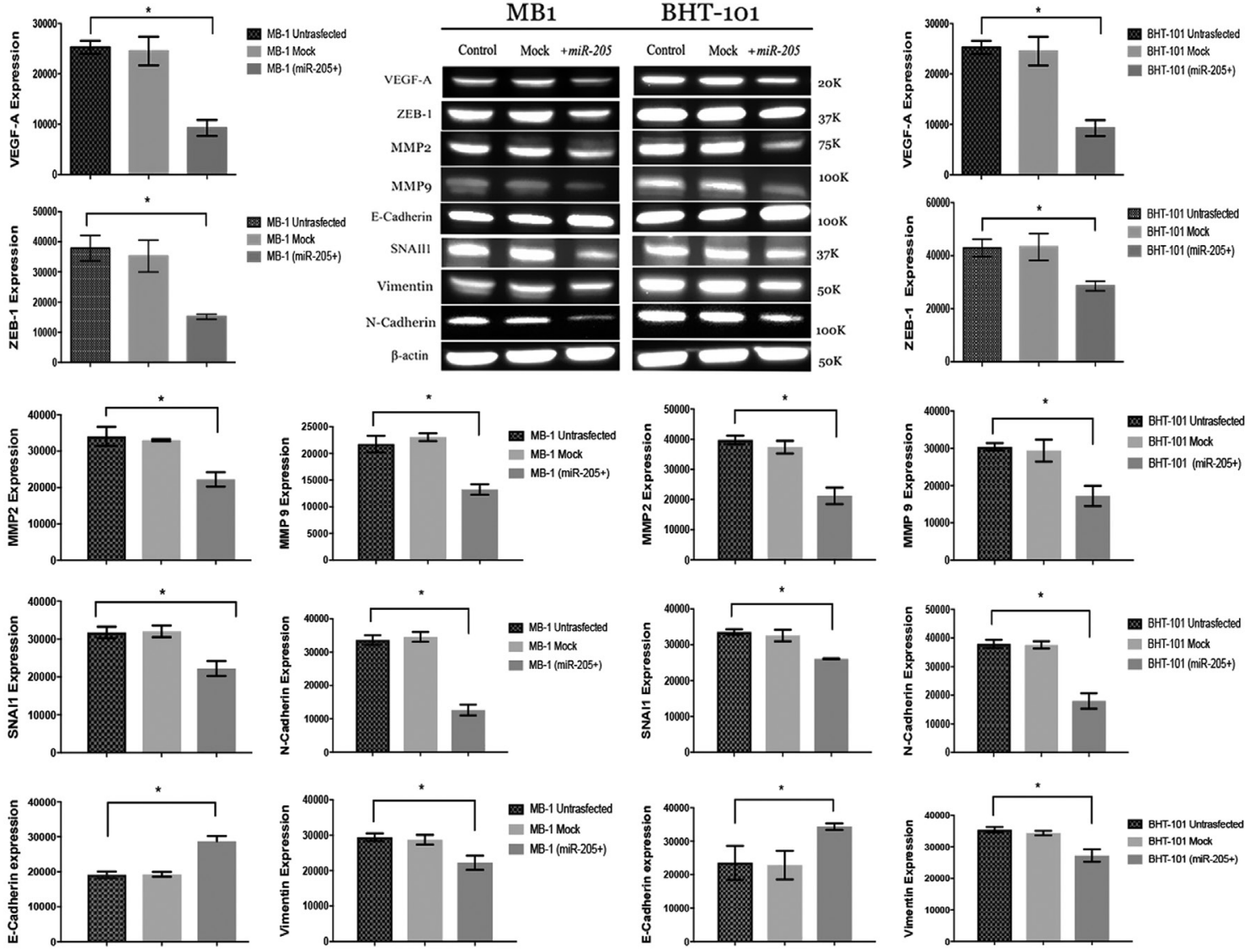

Figure 2

(A and B) Normalised relative expression of mir-205 by qRT-PCR to confirm overexpression of miR-205 after permanent transfection of anaplastic thyroid carcinoma cells (MB-1 and BHT-101) with pCMV-MIR-205 vector $(P<0.05)$. (C and D) Protein levels of VEGFA, ZEB1, MMP2, MMP9, E-cadherin and SNAI1, vimentin and $\mathrm{N}$-cadherin were evaluated by Western blot. There was significant downregulation of VEGF-A, ZEB1, MMP2, MMP9, SNAI1, vimentin and $\mathrm{N}$-cadherin and elevated expression of E-cadherin because of overexpression of miR-205 in both transfected cancer cells (MB1 and BHT-101) compared to negative controls. $\beta$-Actin was used as a housekeeping control $(P<0.05)$.

major angiogenic growth factor in cancer cells, as well as MMP2 and MMP9 as the proposed downstream products involved in angiogenesis (Fig. 2C and D). The pCMV-MIR205-transfected MB-1 and BHT-101 thyroid carcinoma cells illustrated a dramatic downregulation of VEGF-A expression $(P<0.05)$ and significant drop in MMP2 and MMP9 $(P<0.05)$.
It was also observed that overexpression of $m i R-205$ led to significant reduction of ZEB1, SNAI1, N-cadherin and vimentin expression in the anaplastic thyroid carcinoma cells (MB-1 and BHT-101) $(P<0.05)$ (Fig. 2C and D). Furthermore, we found that E-cadherin expression had been upregulated after miR-205 transfection in both anaplastic thyroid carcinomas when compared to 
pCMV-MIR mock and untransfected cancer cells $(P<0.05)$. These results indicate that $m i R-205$ could act as an antiangiogenic agent to target VEGF-A hence affecting MMP2 and MMP9. As a result of the MMP family's role in the process of EMT, miR-205 could also regulate invasive and metastatic behaviour of a malignant lesion by significantly manipulating the expression of ZEB1, SNAI1, N-cadherin, vimentin and E-cadherin.

\section{miR-205 profoundly suppresses human VEGF-A secretion and endothelial tube formation ability}

Taking advantage of the Human VEGFA-165 ELISA kit, we determined the inhibitory effect of $m i R-205$ expression on VEGF-A production in anaplastic thyroid carcinoma cells. Following three days of incubation of MB-1 and BHT-101 pCMV-MIR-205-transfected cells, pCMV-MIR mock and untransfected cells in hypoxic conditions, the expression of soluble forms of VEGF-A was gradually reduced by
$36 \%$ and 30\%, in the pCMV-MIR-205-transfected cells, respectively $(P<0.05)$. This indicates that permanent miR-205-transfected cancer cells released significantly lower levels of VEGF-A into culture media when compared to control cell lines (Fig. 3A).

Given that our previous data shows miR-205 can target VEGF-A (Salajegheh et al. 2015) and following the significant reduction of VEGF-A secretion in MB-1 and BHT-101 thyroid carcinoma cells, we further determined a potentially anti-angiogenic role of miR-205 using a co-culture system and HUVEC tube formation assay in vitro. As shown in Fig. 3B, depletion of VEGF-A expression as a result of stable transfection of the miR-205 precursor in thyroid carcinoma cell lines significantly repressed HUVEC tube formation ability when compared with mock transfected and untransfected cancer cell lines $(P<0.05)$. A $42 \%$ and $49 \%$ decrease in total loop numbers were observed in miR-205-transfected MB-1 and BHT-101 cells,
A

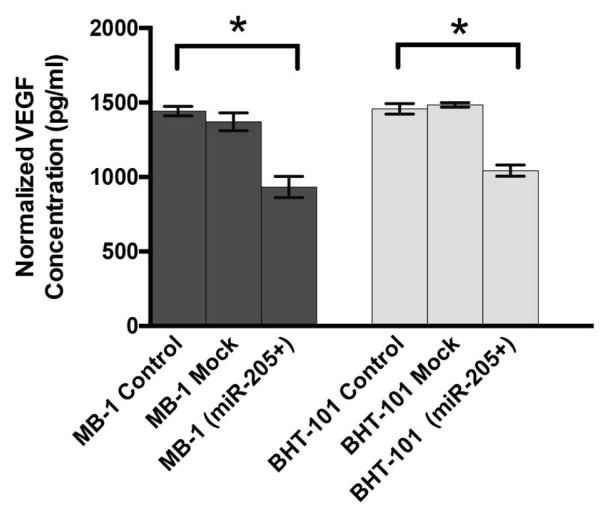

B

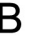

MB-1

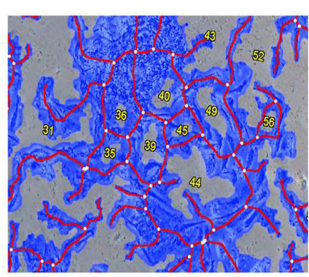

BHT-101

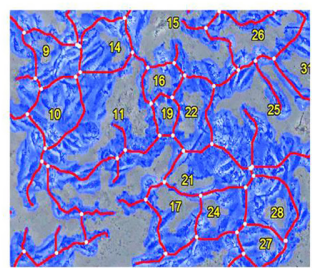

Mock
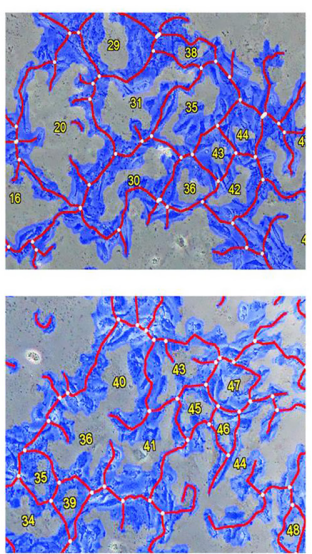

miR-205 transfected
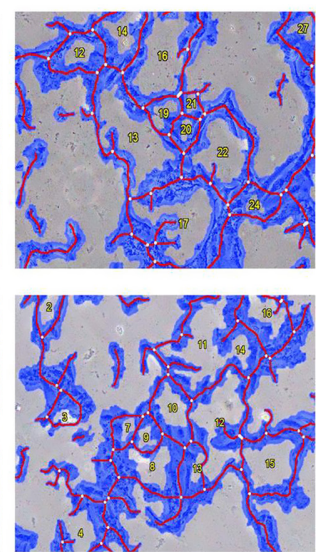

C

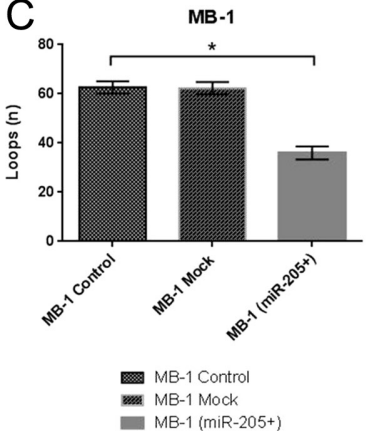

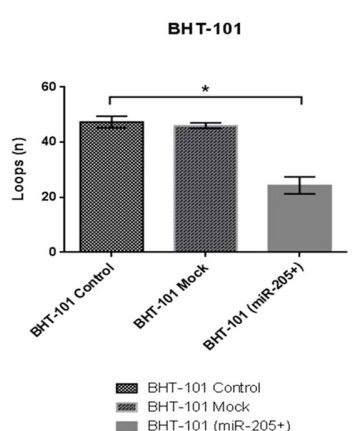

BHT-101 Mock

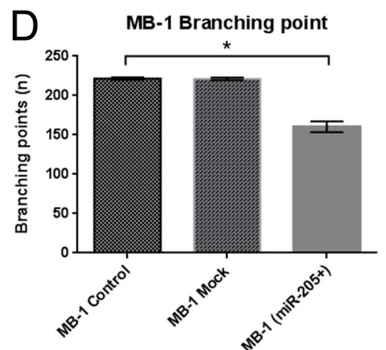

MB-1 Control $=$ MB-1 Mock

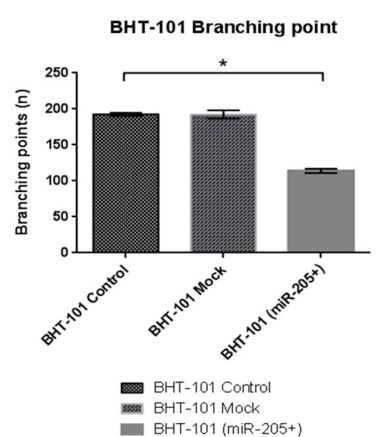

Figure 3

Upregulation of miR-205 diminishes VEGFA secretion and endothelial tube formation. (A) Ectopic expression of miR-205 significantly reduced secreted levels of VEGFA in miR-205-transfected MB-1 and BHT-101 cancer cells culture media collected after 72-h incubation in hypoxic condition ( $P<0.05)$. (B) HUVEC endotube formation decreased in the presence of miR-205 expressing MB-1 and BHT-101 cancer cells compared to untransfected and mock cancer cells. (C and D) Quantitative analysis of the morphological tube formation parameters was evaluated. Total loop numbers and branching points were significantly reduced in HUVECs co-cultured with miR-205 expressing MB-1 and BHT-101 cancer cells compared to untreated cancer cells $(P<0.05)$. 
respectively, when compared to mock transfected and untransfected controls. Similar significant trends were also noticed in the number of branching points. Co-cultured HUVECs with the two anaplastic thyroid carcinoma cell lines (MB-1 and BHT-101) overexpressing miR-205 showed significantly fewer branching points in comparison with HUVECs co-cultured with mock transfected groups and untransfected cancer cell lines $(P<0.05)$ (Fig. 3C and D).

\section{Reduction of migration ability of cancer cells by $\operatorname{miR}-205$}

The effect of miR-205 on tumour migration was assessed and quantified using a scratch wound-healing assay. Cells transfected with miR-205 as well as the control cancer cells were seeded on 6-well plates and grown in standard complete growth media to reach $80 \%$ confluence. After making a scratch on each confluent cell monolayer and replacing the complete media with 5\% FBS-media, the open wound area was monitored for three days. The results demonstrated that miR-205 could noticeably suppress cancer cell mobility and migration ability in MB-1 and BHT-101 when compared to controls $(P<0.05)$ (Fig. 4A, B, C and D).

Due to a significant drop of MB-1 and BHT-101 cancer cell proliferation in ideal conditions (20\% FBS) in the presence of miR-205 (Salajegheh et al. 2015), it was considered that the cancer cell migratory behaviour in 5\% FBS condition was solely a result of their invasive characteristics and any inevitable proliferation would be minor and could be ignored. MB-1 and BHT-101 cancer cells could not survive in condition below 5\% for this test.

\section{Permanent transfection of $\mathbf{m i R - 2 0 5}$ inhibits the invasive characteristics of cancer cells}

As invasion through the extracellular matrix is a vital stage in tumour metastasis, the invasiveness of cancer cells was evaluated using a transwell chamber invasion assay. For this purpose, we cultured cancer cells on transwell inserts with 8 - $\mu \mathrm{m}$ pore size and illustrated the anti-invasive role of miR-205 in cancer cells. As shown in Fig. 4E and G, overexpression of miR-205 noticeably attenuates the number of invading pCMV-MIR-205-transfected cells compared with pCMV-MIR mock and untransfected cells in MB-1 and BHT-101 cancer cell lines $(P<0.05)$ (Fig. 4F and $\mathrm{H}$ ).

\section{Elevated expression of $\mathbf{m i R}-205$ suppresses angiogenesis, invasion and tumour growth in vivo}

We investigated whether ectopic expression of miR-205 in anaplastic thyroid carcinoma cells affects tumour angiogenesis, growth and EMT in vivo. MB-1 and BHT-101 cancer cells stably overexpressing miR-205 showed slower growth compared with controls. In the four weeks after injection, animals with miR-205 expressing tumour showed approximately $40 \%$ smaller volume of the tumour compared with controls $(P<0.05)$ (Fig. 5A, B, $\mathrm{C}, \mathrm{D}$ and $\mathrm{E})$. At the end point of the in vivo experiment, the weight of removed lesions was measured. MB-1 cancer lesions with pCMV-MIR-205 showed a significant 50\% weight reduction and BHT-101 lesions overexpressing miR-205 presented with a $55 \%$ lower weight in comparison to control and mock groups $(P<0.05)$ (Fig. 5 F).

To explore the tumour suppressive role of $m i R-205$ in angiogenesis, growth and EMT/invasion in vivo, cancer tissues in the mice were examined for morphological changes and immunohistochemical analysis (Figs 6 and 7). The cancers derived from MB-1 and BHT-101 transfected with miR-205 were often of epithelioid morphology and had less mitotic/necrotic features and spindle-shaped appearance when compared with mock transfected and control tissues (Fig. 6A). Initiation of a MET event (mesenchymal epithelial transition) occurs when undifferentiated malignant cell transforms towards a differentiated phenotype, obtaining epithelial cell characteristics. This was investigated by examining the expression of a marker of thyroid differentiation (TTF1). Although no significant difference could be established, a raising trend of focal TTF1 expression was noticed in both MB-1 and BHT-101 lesions transfected with miR-205) (Fig. 6B). Both MB-1 and BHT-101 lesions transfected with $m i R-205$ showed an approximately 52\% drop in nuclear staining of Ki67 $(P<0.05)$ (Fig. 6C), which is in concordance with lower tumour weight results presented previously.

Significant downregulation of CD34 as evidence of reduced vascularisation in transfected tumour xenografts confirmed the anti-angiogenic potential of miR-205 $(P<0.05)$ (Fig. 7A). This result exhibited a significantly reduced microvascular density (MVD) in miR-205 transfected lesions compared to controls $(P<0.05)$ (Fig. 7A graphs). Additionally, a comparison of the E-cadherin and vimentin staining in tumour xenografts showed significantly higher expression of E-cadherin and lower expression of vimentin in those tumours overexpressing miR-205 overexpression $(P<0.05)$ (Fig. 7B and C). 

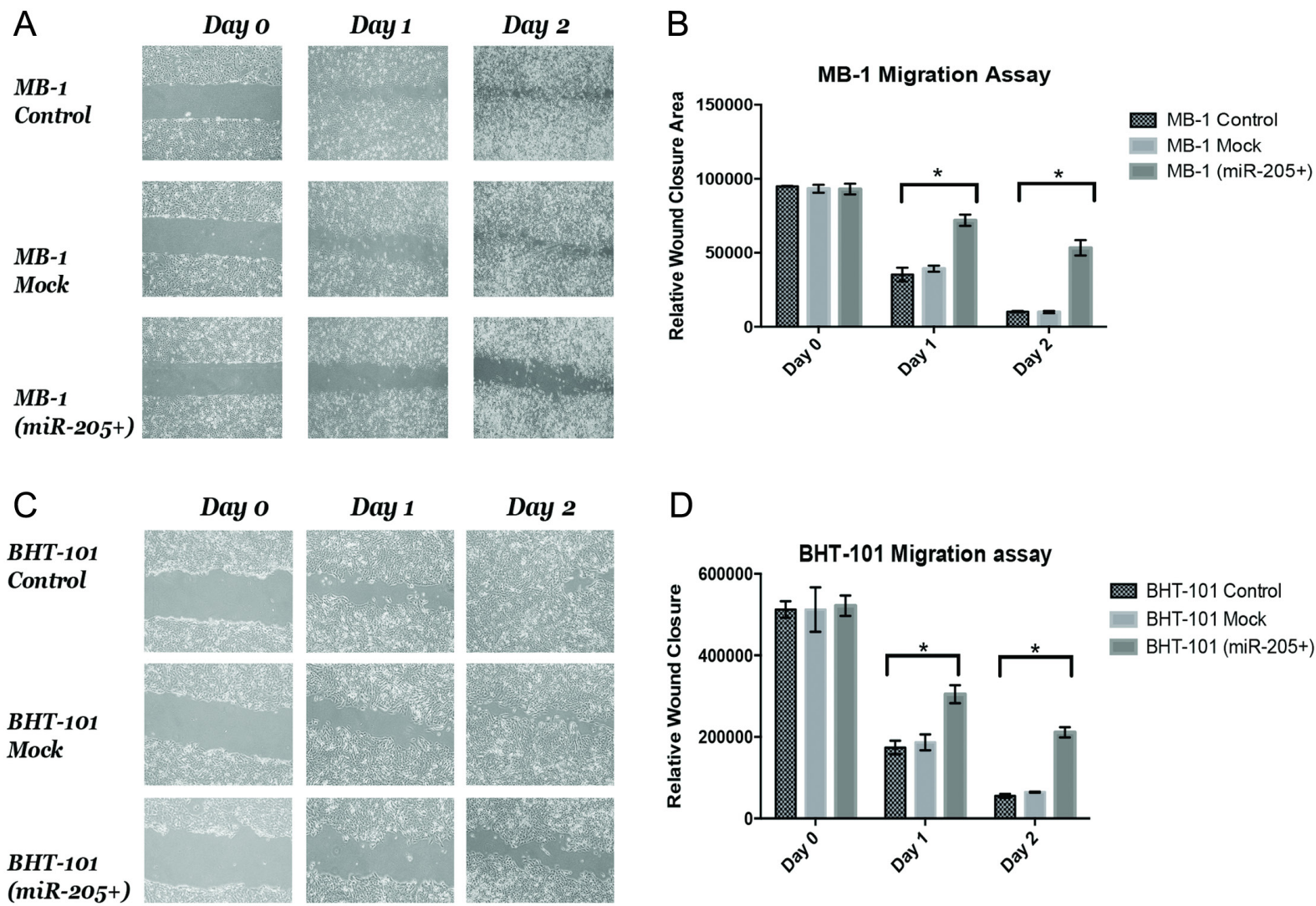

$\left(\operatorname{miR}-205^{+}\right)$
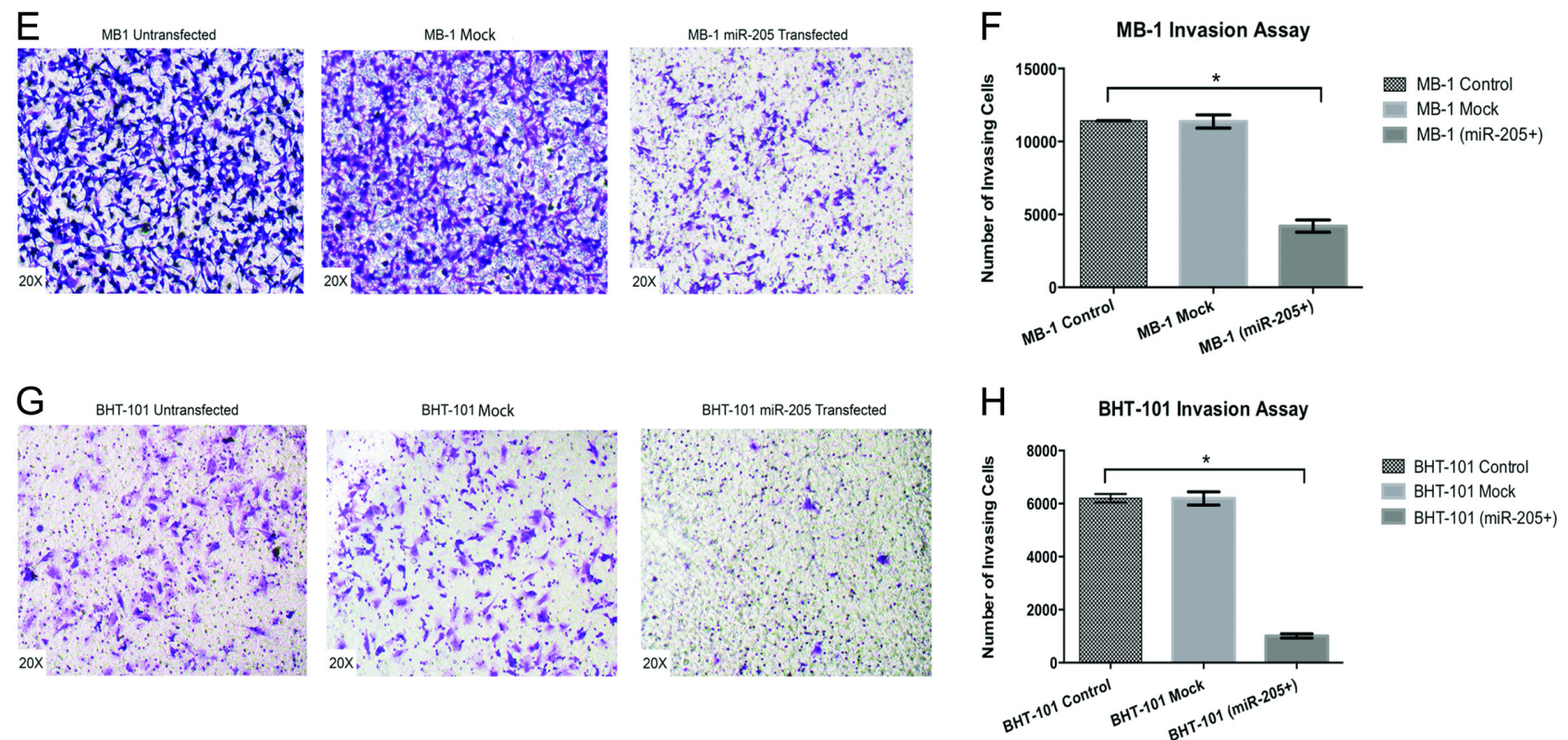

Figure 4

Overexpression of miR-205 inhibits invasive characteristics of anaplastic thyroid carcinoma cells. (A, B, C and D) Wound-healing assay showed that elevated expression of miR-205 significantly hindered wound closure area over three days in MB-1 and BHT-101 $(P<0.05)$. $(E, F, G$ and $H)$ Invasion assay was utilised to confirm anti-invasive role of miR-205. Ectopic expression of miR-205 in transfected MB-1 and BHT-101 cells significantly inhibited the number of invading cells when compared with control cells $(P<0.05)$. 
A

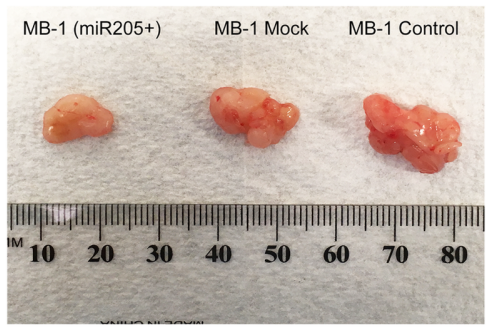

D

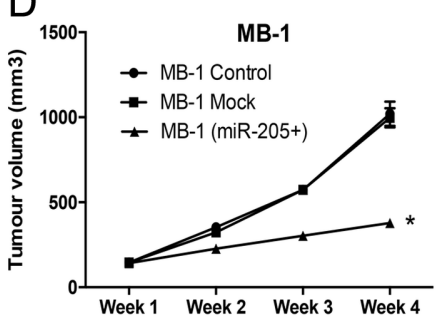

B

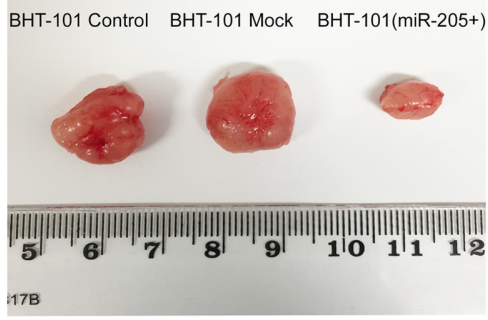

E

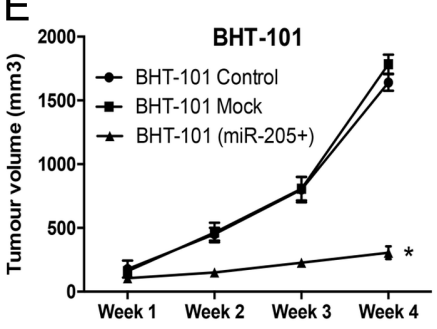

C

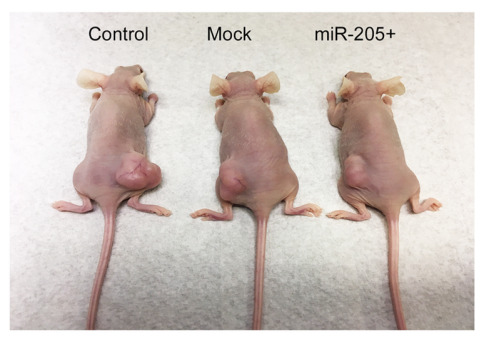

$\mathrm{F}$

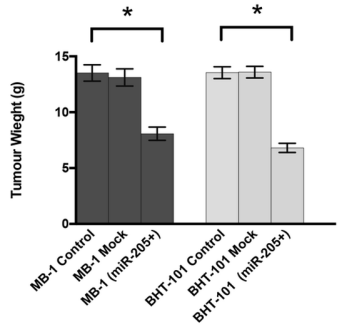

Figure 5

miR-205 impedes the growth of MB-1 and BHT-101 thyroid tumour in vivo. (A and B) Comparison of samples of MB-1and BHT-101 miR-205-transfected, mock and control xenografts are shown. (C) Samples of implanted xenografts after 28 days of proliferation. Differences in lesions' sizes and vascularised appearance were noticeable in miR-205-treated and control groups. (D and E) Lesions' volumes were measured in mm ${ }^{3}$ over time (4 weeks). Significant reduction of tumour volume was noted in both miR-205-transfected xenografts. (F) A noticeable drop in average tumour weight of miR-205-expressing thyroid carcinoma cells (MB-1 and BHT-101) was noted in comparison with mock and control cells $(P<0.05)$.

\section{Discussion}

The leading cause of mortality in patients with anaplastic thyroid carcinoma is linked to its profound metastatic and invasive characteristics (Guo et al. 2014). Therefore, this study investigated the simultaneous suppression of angiogenesis, proliferation and invasion of this aggressive tumour through microRNA expression, which could be utilised for cancer treatment.

Our previous findings indicated that the level of $m i R-205$ expression is notably downregulated in papillary thyroid carcinoma and anaplastic thyroid carcinoma when compared to non-neoplastic thyroid tissues. Located in chromosome 1q32.2, microRNA-205 (miR-205) is one of the well-studied miRNAs, which can be over-expressed or downregulated in different cancers, based on the cell type and its targets. Its dual function as a tumour suppressor or an oncogene suggests that $m i R$-205 could have an effective role in the induction or repression of tumorigenesis through regulation of numerous signalling pathways such as angiogenesis, cancer cell apoptosis, proliferation and EMT (Vosgha et al. 2014, Salajegheh et al. 2015).

Various genes can be targeted by miR-205 including VEGFA, ZEB1, BCL2 (B-cell lymphoma 2), E2F1 (E2F transcription factor 1) and HER2 (receptor tyrosine-protein kinase-2), which can modulate many cellular mechanisms (Vosgha et al. 2014). miRNAs only need to be partially complementary to their targets to regulate their activity as a result of imperfect base-pairing. Therefore, our research utilised this property of $m i R-205$ to halt angiogenesis and EMT of anaplastic thyroid carcinoma by targeting VEGFA and ZEB1.

VEGF-A is an essential inducer of tumour angiogenesis in thyroid carcinoma (Salajegheh et al. 2011, 2013). Overexpression of VEGF-A and its receptor have been reported in a variety of tumours such as thyroid, skin, breast, lung and oesophageal cancers, etc. (Salajegheh et al. 2011, 2016, Das \& Wakelee 2012, Rajabi et al. 2012, $\mathrm{Xu}$ et al. 2015). In this pathway of tumour angiogenesis and in the downstream of VEGF, many proteins such as MMP2 and MMP9 have been reported to be involved in the enhancement and expansion of neo-angiogenesis process in different malignant lesions (Turner et al. 2003, Lin et al. 2015, Mahecha \& Wang 2017). Interaction of VEGF and MMPs have been reported in lung cancer, endometrial cancer and breast cancer (Radisky \& Radisky 2010, Lin et al. 2015, Mahecha \& Wang 2017). Likewise, in this study, we noticed that, following the inhibition of VEGF in anaplastic thyroid carcinoma, expression of MMP2 and MMP9 were significantly reduced. 
A Morphological Features

Untransfected Control

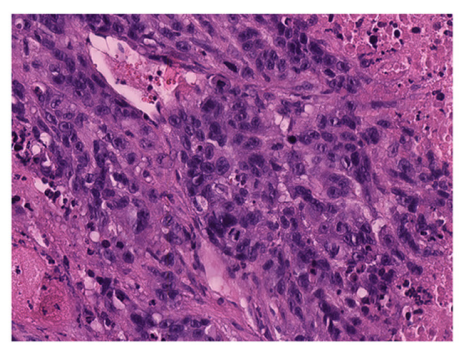

Mock Control

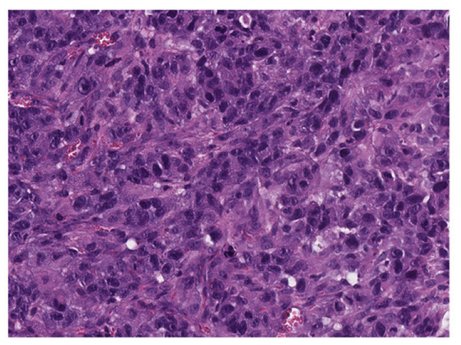

miR-205+ Transfected

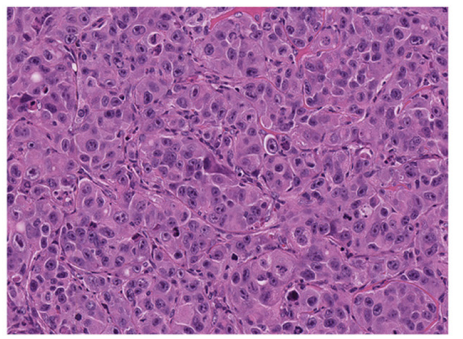

B

TTF1
Untransfected

BHT-101

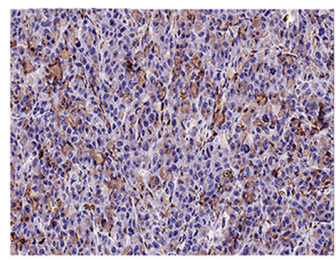

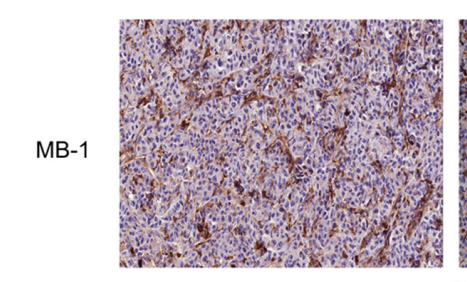

Mock
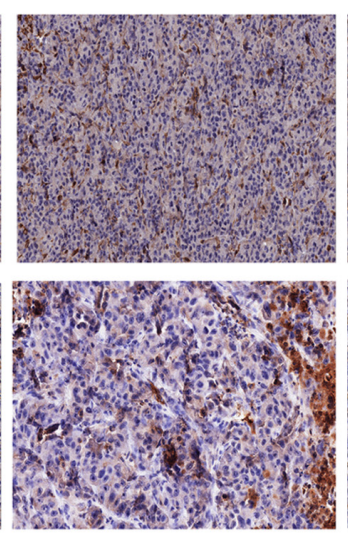
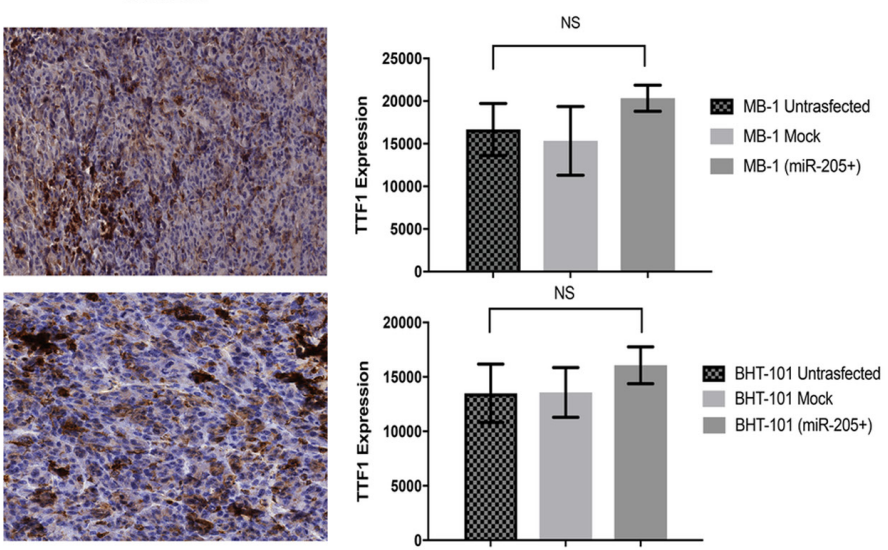

C $\quad \mathrm{Ki}-67$

Untransfected
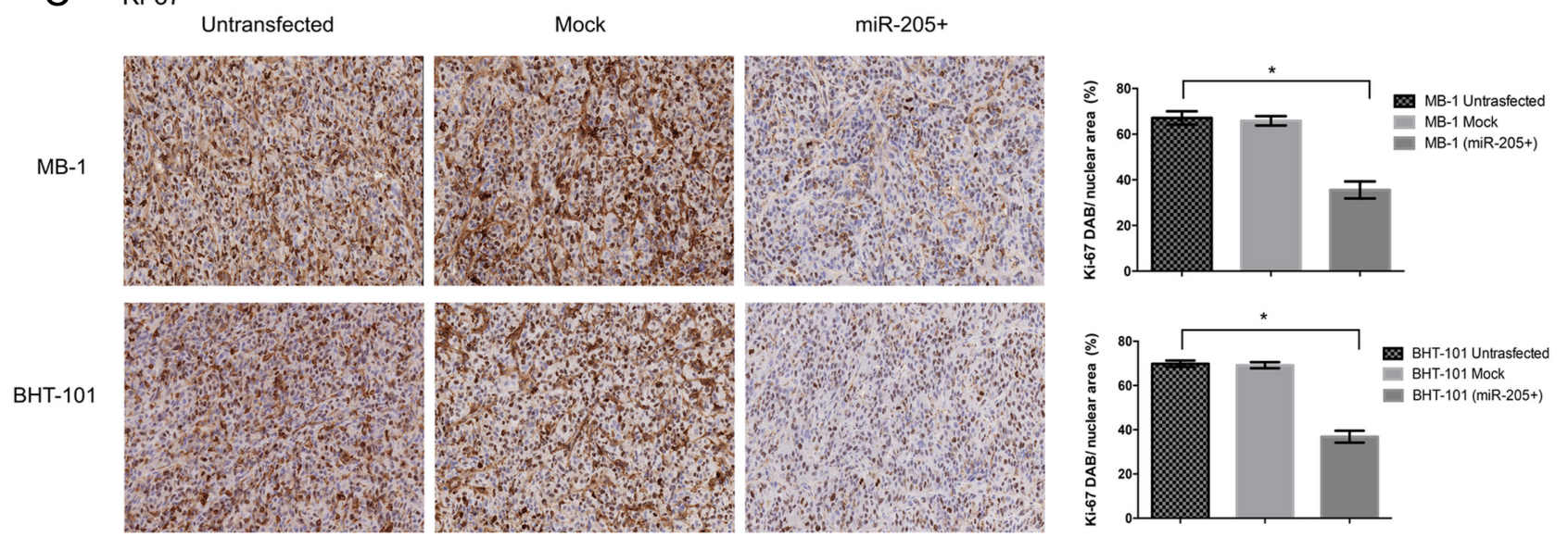

\section{Figure 6}

Histological appearance of tumour xenograft in different experiments and a study of immunological markers for differentiation and proliferation index. (A) Representative histological appearance of tumour xenograft transfected with miR-205 compared to mock and untransfected control. The cancer lesions from control and mock mice reveal anaplastic carcinoma with more frequent mitotic figures, prominent nuclear pleomorphism and spindle cell morphology, whereas transfected tumour xenografts show anaplastic carcinoma with more organoid arrangement of tumour cells with epithelioid morphology. (B) Focal overexpression of thyroid transcription factor 1 (TTF1) in miR-205-transfected MB-1 and BHT-101 xenografts reflects the potential role of miR-205 in forcing the undifferentiated cancer cell to shift towards more epithelial appearance and characteristics, leading to reduction of EMT and invasive morphology in anaplastic thyroid carcinoma. (C) Significant reduction of Ki-67 expression, as a proliferation marker in MB-1- and BHT-101expressing miR-205, showed the tumour suppressive role of miR-205 in thyroid tumour xenografts. Magnification: $20 \times$; NS, not significant; (*) significant.

Although there are many modalities of therapies including surgery, chemotherapy, radiation therapy and combination treatments available in the treatment of anaplastic thyroid carcinoma, the majority of patients with anaplastic thyroid carcinoma survive for only a few months, and their survival rates have not significantly 
A $\quad$ CD-34
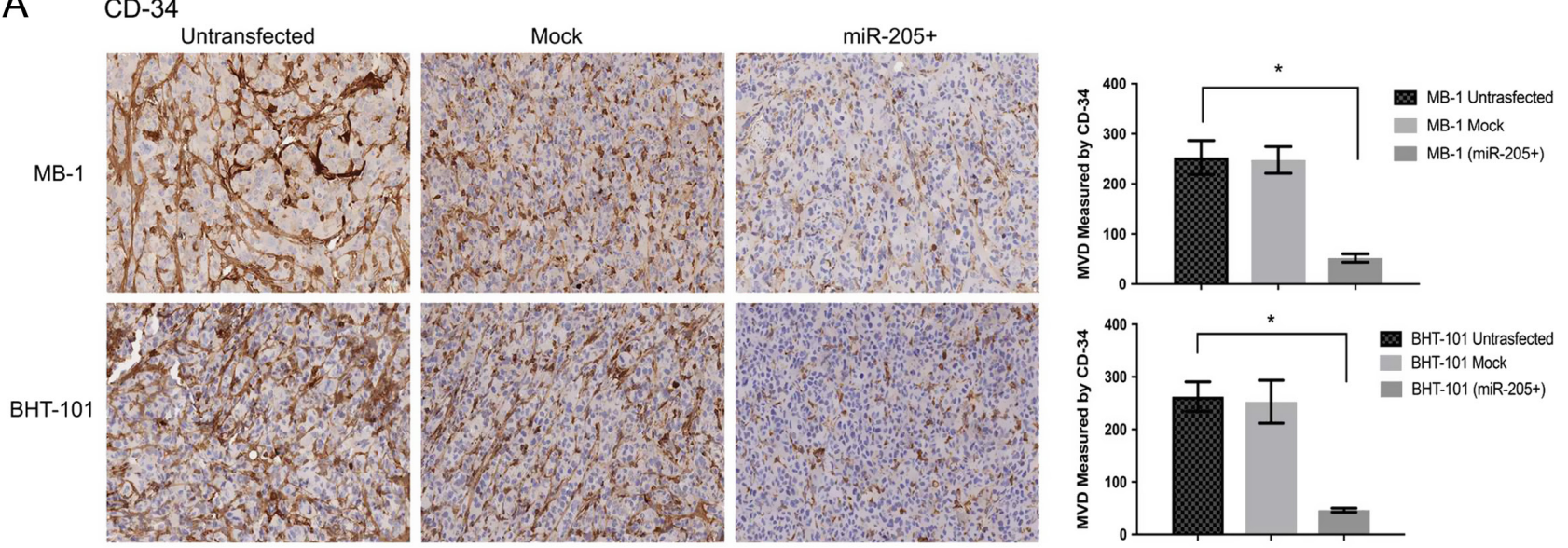

B E-Cadherin

\section{Untransfected}
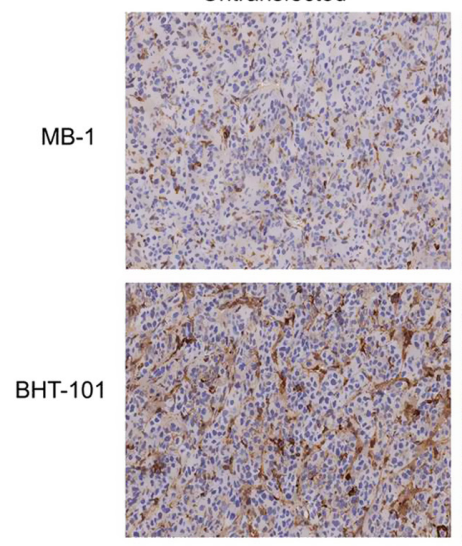

C Vimentin

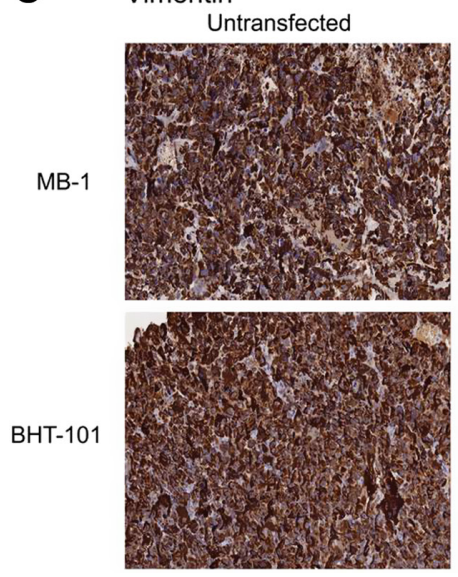

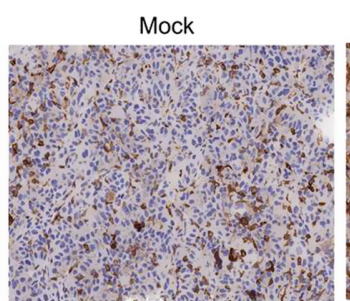
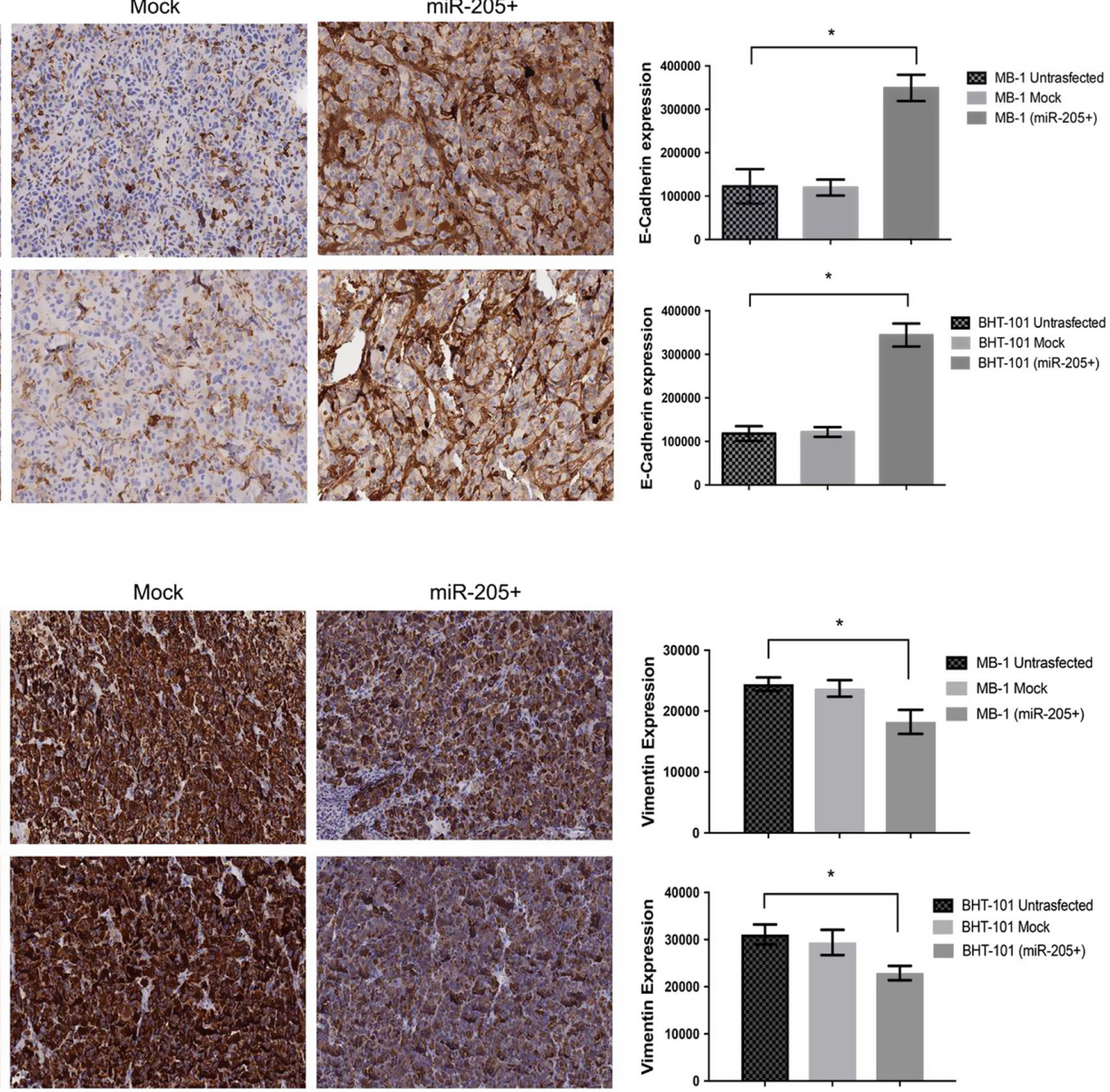

Figure 7

High level of miR-205 is associated with less angiogenic and invasive properties of anaplastic thyroid carcinoma in vivo. (A) Immunostaining of CD34 indicated significant reduction of micro-vessel density (MVD) in miR-205 expressing MB-1and BHT-101 xenografts compared to mock and control xenografts. (B and C) Significant expression changes of E-cadherin and vimentin in miR-205-transfected MB-1and BHT-101 xenografts reflected the role of miR205 in the reduction of EMT and invasive morphology in anaplastic thyroid carcinoma. Magnification: 20x; (*) significant.

changed over time (Smith \& Nucera 2015). Unsurprisingly, anaplastic thyroid carcinomas often show drug resistance to conventional therapies (Harris \& Bible 2011). Due to the highly angiogenic and lymphangiogenic characteristic of thyroid carcinoma, there have been several targeted therapy approaches proposed to hinder angiogenesis 
and metastasis mechanisms, specifically. Anti-angiogenic drugs, such as bevacizumab and sorafenib, are not able to significantly reduce the progression of anaplastic thyroid carcinoma. They also have several side effects including cardiovascular toxicity and dyspnoea, which make their use less desirable (Smith \& Nucera 2015).

In this study, we have shown that miR-205 acts as one of the key regulators of multiple neoplastic mechanisms in anaplastic thyroid carcinoma. This was evidenced when permanent overexpression of miR-205 resulted in significant reduction in production of VEGF-A, a noticeable drop in vascular formation in vitro and microvascular density in vivo. Similar results showing the role of miR-205 in targeting VEGF-A and angiogenesis have been reported previously in other cancers (Yue et al. 2012, Hu et al. 2016). miR-205 is potentially able to reduce the speed of proliferation and growth in different thyroid cancer cell lines (Salajegheh et al. 2015). In this study, we further validated and enhanced those results, demonstrating a significant reduction in the size and weight of anaplastic thyroid carcinoma in tumour xenografts derived from miR-205 expressing stable cell lines. Therefore, $m i R-205$ induction or introduction could be a promising anti-angiogenic and anti-proliferative strategy in the management of patients with anaplastic thyroid carcinoma.

Angiogenesis is one of the crucial stages of tumour metastasis and correlates with aggressiveness and invasiveness in thyroid carcinoma (Salajegheh et al. 2015, 2016). Tumour metastasis is an angiogenesis-dependent mechanism and any strategy to block angiogenesis could be a potential method to stop cancer progression (Ferrara \& Kerbel 2005). In the current study, we linked miR-205 to the invasive behaviour of anaplastic thyroid carcinoma in two different cell lines. First, we confirmed a significant reduction in the migratory abilities of anaplastic thyroid carcinoma in miR-205 stable cell lines compared to control cells. This result was further supported by performing invasion assays, indicating a decreased number of invasive cells transferred through transwell membranes due to miR-205 overexpression. These data are consistent with previous studies showing an anti-invasive and antimigratory role of miR-205 in breast and colon cancer (Wu et al. 2009, Li et al. 2015b).

Numerous studies suggest that EMT is strongly associated with thyroid carcinoma progression and metastasis (Buehler et al. 2013, Hardin et al. 2013, Montemayor-Garcia et al. 2013, Fantozzi et al. 2014, Guo et al. 2014). EMT, as an initiator of cancer metastasis, is closely linked to downregulation of E-cadherin, which is a key cell-cell adhesion molecule, and the simultaneous upregulation of several transcription factors including ZEB1, ZEB2, SNAI1, Slug, vimentin and several MMPs (De Craene \& Berx 2013). It has been reported that anaplastic thyroid carcinoma showed reduced expression of E-cadherin and a high expression of ZEB1, which is connected to the invasiveness and metastatic properties of anaplastic thyroid carcinoma (Montemayor-Garcia et al. 2013).

To further investigate the inhibitory function of $m i R-205$ in the migration and invasion of anaplastic thyroid carcinoma, the expression of ZEB1 (a target of miR-205) and consequently its downstream-interacting proteins such as E-cadherin, $\mathrm{N}$-cadherin, SNAI1 and vimentin were assessed and compared in miR-205 transfected and control cell lines. Those transfected anaplastic thyroid carcinoma cells overexpressing miR-205 showed significant reduction of ZEB1, SNAI1, vimentin and N-cadherin and increased expression of membranous E-cadherin when compared to control anaplastic carcinoma cells. A significant antiinvasion role of miR-205 in anaplastic thyroid carcinoma was also observed in the migration assay and invasion assay experiments. These observations could be associated with the reduction of EMT markers (ZEB1, SNAIl, vimentin and $\mathrm{N}$-cadherin) in addition to inhibition of MMP2 and MMP9, following the VEGF drop, and the increase of the adhesion molecule, E-cadherin, that we have seen previously. Further evidence was added from in vivo studies, where tumours expressing miR-205 showed reduced invasive characteristics by presenting with less vascularisation, lower proliferation and more cell-tocell adhesion due to increased membranous E-cadherin. Finally, those tumours had more epithelioid morphology, less mitotic/necrotic features and less spindle-shaped appearance.

Due to the limitations of the xenograft study model, cancer cells implanted in mice are not highly metastatic. Thus, injection of cell lines into the blood stream seems to be unrealistic or unrepresentative to the nature and morphological needs of real cancer metastasis (Holliday \& Speirs 2011). Likewise, neither MB-1 nor BHT-101 with highly invasive characteristics showed any metastasis to distant sites. This suggests that a shift of transition is taking place, due to an increase of $m i R-205$ and decrease of ZEB1 and other EMT markers, where the mesenchymal morphology is changing towards an epithelial pattern (MET). These findings are in concordance with previous studies in breast cancer where downregulation of miR-205 was correlated with overexpression of ZEB1/2 and poor survival of the patient (Gregory et al. 2008, Zhang et al. 2014). 
In conclusion, our data demonstrated for the first time that elevated miR-205 regulates two hallmarks of anaplastic thyroid carcinoma, angiogenesis and EMT, by targeting VEGFA and ZEB1 in vitro and in vivo. The high level of miR-205 is associated with less biologically aggressive features of anaplastic thyroid carcinoma, suppressing thyroid cancer cell growth and invasion. Hence, our functional analysis of miR-205 suggests that targeting multiple pathways via partial matches between miR-205 and its targets can be employed for the development of a miR-205-based therapeutic agent against angiogenesis and EMT in anaplastic thyroid carcinoma.

\section{Declaration of interest}

The authors declare that there is no conflict of interest that could be perceived as prejudicing the impartiality of the research reported.

\section{Funding}

This work did not receive any specific grant from any funding agency in the public, commercial or not-for-profit sector.

\section{Author contribution statement}

$A A$ and $A L$ conceived the idea of the study; $H V$ and $A A$ designed and ran experiments and wrote the manuscript; $H V, A A, R S$ and $A L$ were involved in the data analysis, interpretation of data and revision of the manuscript.

\section{Acknowledgements}

The authors would like to thank the funding support of student scholarships from Griffith University, the project grants of the Menzies Health Institute of Queensland from Griffith University as well as the Smart State Fellowship from the Queensland Government. In addition, they would like to thank Melissa Leung for her help in the imaging of the histopathology work and Dr Kais Kasem for the help with immunostaining. Furthermore, they would like acknowledging the staff in Helix Pathology and NH Diagnostics for their invaluable support in the histological work of the animal experiment.

\section{References}

Alfano RW, Leppla SH, Liu S, Bugge TH, Ortiz JM, Lairmore TC, Duesbery NS, Mitchell IC, Nwariaku F \& Frankel AE 2010 Inhibition of tumor angiogenesis by the matrix metalloproteinase-activated anthrax lethal toxin in an orthotopic model of anaplastic thyroid carcinoma. Molecular Cancer Therapeutics 9 190-201. (https://doi. org/10.1158/1535-7163.MCT-09-0694)

Araujo RF Jr, Lira GA, Vilaca JA, Guedes HG, Leitao MC, Lucena HF \& Ramos CC 2015 Prognostic and diagnostic implications of MMP-2, MMP-9, and VEGF-alpha expressions in colorectal cancer. Pathology: Research and Practice 211 71-77. (https://doi.org/10.1016/j. prp.2014.09.007)

Braun J, Hoang-Vu C, Dralle H \& Huttelmaier S 2010 Downregulation of microRNAs directs the EMT and invasive potential of anaplastic thyroid carcinomas. Oncogene 29 4237-4244. (https://doi. org/10.1038/onc.2010.169)

Buehler D, Hardin H, Shan W, Montemayor-Garcia C, Rush PS, Asioli S, Chen H \& Lloyd RV 2013 Expression of epithelial-mesenchymal transition regulators SNAI2 and TWIST1 in thyroid carcinomas. Modern Pathology 26 54-61. (https://doi.org/10.1038/ modpathol.2012.137)

Chruscik A \& Lam AK 2015 Clinical pathological impacts of microRNAs in papillary thyroid carcinoma: a crucial review. Experimental and Molecular Pathology 99 393-398. (https://doi.org/10.1016/j. yexmp.2015.08.013)

Das M \& Wakelee H 2012 Targeting VEGF in lung cancer. Expert Opinion on Therapeutic Targets 16 395-406. (https://doi.org/10.1517/14728222 .2012.669752)

De Craene B \& Berx G 2013 Regulatory networks defining EMT during cancer initiation and progression. Nature Reviews Cancer 13 97-110. (https://doi.org/10.1038/nrc3447)

Fantozzi A, Gruber DC, Pisarsky L, Heck C, Kunita A, Yilmaz M, MeyerSchaller N, Cornille K, Hopfer U, Bentires-Alj M, et al. 2014 VEGFmediated angiogenesis links EMT-induced cancer stemness to tumor initiation. Cancer Research 74 1566-1575. (https://doi. org/10.1158/0008-5472.CAN-13-1641)

Ferrara N \& Kerbel RS 2005 Angiogenesis as a therapeutic target. Nature 438 967-974. (https://doi.org/10.1038/nature04483)

Folkman J \& Shing Y 1992 Angiogenesis. Journal of Biological Chemistry 267 10931-10934.

Giatromanolaki A, Lyberakidis G, Lyratzopoulos N, Koukourakis MI, Sivridis E \& Manolas C 2010 Angiogenesis and angiogenic factor expression in thyroid cancer. Journal of BUON 15 357-361.

Gregory PA, Bert AG, Paterson EL, Barry SC, Tsykin A, Farshid G, Vadas MA, Khew-Goodall Y \& Goodall GJ 2008 The miR-200 family and miR-205 regulate epithelial to mesenchymal transition by targeting ZEB1 and SIP1. Nature Cell Biology 10 593-601. (https://doi. org/10.1038/ncb1722)

Guo Z, Hardin H \& Lloyd RV 2014 Cancer stem-like cells and thyroid cancer. Endocrine-Related Cancer 21 T285-T300. (https://doi. org/10.1530/ERC-14-0002)

Hardin H, Montemayor-Garcia C \& Lloyd RV 2013 Thyroid cancer stemlike cells and epithelial-mesenchymal transition in thyroid cancers. Human Pathology 44 1707-1713. (https://doi.org/10.1016/j. humpath.2013.01.009)

Harris PJ \& Bible KC 2011 Emerging therapeutics for advanced thyroid malignancies: rationale and targeted approaches. Expert Opinion on Investigational Drugs 20 1357-1375. (https://doi.org/10.1517/1354378 4.2011.614230)

Hoeben A, Landuyt B, Highley MS, Wildiers H, Van Oosterom AT \& De Bruijn EA 2004 Vascular endothelial growth factor and angiogenesis. Pharmacological Reviews 56 549-580. (https://doi.org/10.1124/ pr.56.4.3)

Holliday DL \& Speirs V 2011 Choosing the right cell line for breast cancer research. Breast Cancer Research 13 215. (https://doi. org/10.1186/bcr2889)

Hu Y, Qiu Y, Yague E, Ji W, Liu J \& Zhang J 2016 miRNA-205 targets VEGFA and FGF2 and regulates resistance to chemotherapeutics in breast cancer. Cell Death and Disease 7 e2291. (https://doi. org/10.1038/cddis.2016.194)

Jia W, Gao XJ, Zhang ZD, Yang ZX \& Zhang G 2013 S100A4 silencing suppresses proliferation, angiogenesis and invasion of thyroid cancer cells through downregulation of MMP-9 and VEGF. European Review for Medical and Pharmacological Sciences 17 1495-1508.

Lam AK 2017 Pathology of endocrine tumors update: World Health Organization new classification 2017 - other thyroid tumors. AJSP: Reviews and Reports 22 209-216. (https://doi.org/10.1097/ PCR.0000000000000183)

Lam KY, Lo CY, Chan KW \& Wan KY 2000 Insular and anaplastic carcinoma of the thyroid: a 45 -year comparative study at a single
2018 Society for Endocrinology Published by Bioscientifica Ltd. Printed in Great Britain 
institution and a review of the significance of p53 and p21. Annals of Surgery 231 329-338. (https://doi.org/10.1097/00000658200003000-00005)

Lamouille S, Xu J \& Derynck R 2014 Molecular mechanisms of epithelial-mesenchymal transition. Nature Reviews Molecular Cell Biology 15 178-196. (https://doi.org/10.1038/nrm3758)

Li J, Li L, Li Z, Gong G, Chen P, Liu H, Wang J, Liu Y \& Wu X 2015a The role of miR-205 in the VEGF-mediated promotion of human ovarian cancer cell invasion. Gynecologic Oncology 137 125-133. (https://doi.org/10.1016/j.ygyno.2015.01.531)

Li P, Xue WJ, Feng Y \& Mao QS 2015b MicroRNA-205 functions as a tumor suppressor in colorectal cancer by targeting cAMP responsive element binding protein 1 (CREB1). American Journal of Translational Research 7 2053-2059.

Lin X, Li HR, Lin XF, Yu ME, Tu XW, Hua ZD, Lin M, Xu NL, Han LL \& Chen YS 2015 Silencing of Livin inhibits tumorigenesis and metastasis via VEGF and MMPs pathway in lung cancer. International Journal of Oncology 47 657-667. (https://doi.org/10.3892/ijo.2015.3058)

Liu L, Tong Q, Liu S, Cui J, Zhang Q, Sun W \& Yang S 2016 ZEB1 upregulates VEGF expression and stimulates angiogenesis in breast cancer. PLOS ONE 11 e0148774. (https://doi.org/10.1371/journal. pone.0148774)

Lo CY, Lam KY \& Wan KY 1999 Anaplastic carcinoma of the thyroid. American Journal of Surgery 177 337-339. (https://doi.org/10.1016/ S0002-9610(99)00052-5)

Mahecha AM \& Wang H 2017 The influence of vascular endothelial growth factor-A and matrix metalloproteinase-2 and -9 in angiogenesis, metastasis, and prognosis of endometrial cancer. OncoTargets and Therapy 10 4617-4624. (https://doi.org/10.2147/ OTT.S132558)

Montemayor-Garcia C, Hardin H, Guo Z, Larrain C, Buehler D, Asioli S, Chen H \& Lloyd RV 2013 The role of epithelial mesenchymal transition markers in thyroid carcinoma progression. Endocrine Pathology 24 206-212. (https://doi.org/10.1007/s12022-013-9272-9)

Qin AY, Zhang XW, Liu L, Yu JP, Li H, Wang SZ, Ren XB \& Cao S 2013 MiR-205 in cancer: an angel or a devil? European Journal of Cell Biology 92 54-60. (https://doi.org/10.1016/j.ejcb.2012.11.002)

Radisky ES \& Radisky DC 2010 Matrix metalloproteinase-induced epithelial-mesenchymal transition in breast cancer. Journal of Mammary Gland Biology and Neoplasia 15 201-212. (https://doi. org/10.1007/s10911-010-9177-x)

Rajabi P, Neshat A, Mokhtari M, Rajabi MA, Eftekhari M \& Tavakoli P 2012 The role of VEGF in melanoma progression. Journal of Research in Medical Sciences 17 534-539.

Salajegheh A, Smith RA, Kasem K, Gopalan V, Nassiri MR, William R \& Lam AK 2011 Single nucleotide polymorphisms and mRNA expression of VEGF-A in papillary thyroid carcinoma: potential markers for aggressive phenotypes. European Journal of Surgical Oncology 37 93-99. (https://doi.org/10.1016/j.ejso.2010.10.010)

Salajegheh A, Pakneshan S, Rahman A, Dolan-Evans E, Zhang S, Kwong E, Gopalan V, Lo CY, Smith RA \& Lam AK 2013 Co-regulatory potential of vascular endothelial growth factor-A and vascular endothelial growth factor-C in thyroid carcinoma. Human Pathology 44 2204-2212. (https://doi.org/10.1016/j. humpath.2013.04.014)

Salajegheh A, Vosgha H, Md Rahman A, Amin M, Smith RA \& Lam AK 2015 Modulatory role of miR-205 in angiogenesis and progression of thyroid cancer. Journal of Molecular Endocrinology 55 183-196. (https://doi.org/10.1530/JME-15-0182)
Salajegheh A, Vosgha H, Rahman MA, Amin M, Smith RA \& Lam AK 2016 Interactive role of miR-126 on VEGF-A and progression of papillary and undifferentiated thyroid carcinoma. Human Pathology 51 75-85. (https://doi.org/10.1016/j.humpath.2015.12.018)

Salnikov AV, Liu L, Platen M, Gladkich J, Salnikova O, Ryschich E, Mattern J, Moldenhauer G, Werner J, Schemmer P, et al. 2012 Hypoxia induces EMT in low and highly aggressive pancreatic tumor cells but only cells with cancer stem cell characteristics acquire pronounced migratory potential. PLOS ONE 7 e46391. (https://doi. org/10.1371/journal.pone.0046391)

Sang QX 1998 Complex role of matrix metalloproteinases in angiogenesis. Cell Research 8 171-177. (https://doi.org/10.1038/ cr.1998.17)

Smith N \& Nucera C 2015 Personalized therapy in patients with anaplastic thyroid cancer: targeting genetic and epigenetic alterations. Journal of Clinical Endocrinology and Metabolism 100 35-42. (https://doi.org/10.1210/jc.2014-2803)

Tsai JH \& Yang J 2013 Epithelial-mesenchymal plasticity in carcinoma metastasis. Genes and Development 27 2192-2206. (https://doi. org/10.1101/gad.225334.113)

Turner HE, Harris AL, Melmed S \& Wass JA 2003 Angiogenesis in endocrine tumors. Endocrine Reviews 24 600-632. (https://doi. org/10.1210/er.2002-0008)

Vosgha H, Salajegheh A, Smith RA \& Lam AK 2014 The important roles of miR-205 in normal physiology, cancers and as a potential therapeutic target. Current Cancer Drug Targets 14 621-637. (https:// doi.org/10.2174/156800961407140926105634)

Wajner SM, Capp C, Brasil BA, Meurer L \& Maia AL 2014 Reduced tissue inhibitor of metalloproteinase-2 expression is associated with advanced medullary thyroid carcinoma. Oncology Letters 7 731-737. (https://doi.org/10.3892/ol.2013.1767)

Workman P, Aboagye EO, Balkwill F, Balmain A, Bruder G, Chaplin DJ, Double JA, Everitt J, Farningham DA, Glennie MJ, et al. 2010 Guidelines for the welfare and use of animals in cancer research. British Journal of Cancer 102 1555-1577. (https://doi.org/10.1038/sj. bjc.6605642)

Wu H, Zhu S \& Mo YY 2009 Suppression of cell growth and invasion by miR-205 in breast cancer. Cell Research 19 439-448. (https://doi. $\operatorname{org} / 10.1038 /$ cr.2009.18)

Xu Y, Brenn T, Brown ER, Doherty V \& Melton DW 2012 Differential expression of microRNAs during melanoma progression: miR-200c, miR-205 and miR-211 are downregulated in melanoma and act as tumour suppressors. British Journal of Cancer 106 553-561. (https:// doi.org/10.1038/bjc.2011.568)

Xu WW, Li B, Lam AKY, Tsao SW, Law SYK, Chan KW, Yuan QJ \& Cheung ALM 2015 Targeting VEGFR1- and VEGFR2-expressing nontumor cells is essential for esophageal cancer therapy. Oncotarget 6 1790-1805. (https://doi.org/10.18632/oncotarget.2781)

Yue X, Wang P, Xu J, Zhu Y, Sun G, Pang Q \& Tao R 2012 MicroRNA-205 functions as a tumor suppressor in human glioblastoma cells by targeting VEGF-A. Oncology Reports 27 1200-1206. (https://doi.org/10.3892/or.2011.1588)

Zhang P, Wang L, Rodriguez-Aguayo C, Yuan Y, Debeb BG, Chen D, Sun Y, You MJ, Liu Y, Dean DC, et al. 2014 miR-205 acts as a tumour radiosensitizer by targeting ZEB1 and Ubc13. Nature Communication 5 5671. (https://doi.org/10.1038/ncomms6671)

van Zijl F, Krupitza G \& Mikulits W 2011 Initial steps of metastasis: cell invasion and endothelial transmigration. Mutation Research $\mathbf{7 2 8}$ 23-34. (https://doi.org/10.1016/j.mrrev.2011.05.002)

Received in final form 20 December 2017

Accepted 9 January 2018

Accepted Preprint published online 9 January 2018 (c) 2018 Society for Endocrinology Published by Bioscientifica Ltd. Printed in Great Britain 